\title{
Light-dependent Oxygen Consumption in Bacteriochlorophyll-Serine-treated Melanoma Tumors: On-line Determination Using a Tissue-inserted Oxygen Microsensor
}

\author{
Judith Zilberstein 1,2, Avraham Bromberg ${ }^{3}$, Anatol Frantz',5, Varda Rosenbach-Belkin², Amnon Kritzmann ${ }^{4}$, \\ Reuven Pfefermann, Yoram Salomon' and Avlgdor Scherz*2 \\ 'Departments of Biological Regulation and 'Biochemistry, The Weizmann Institute of Science, Rehovot, Israel; \\ 3Department of Physical Chemistry, Israel Institute for Biological Research, Ness-Ziona, Israel; \\ ${ }^{4}$ Optical Sensor Technologies (OST), Kiryat Bialik, Israel and \\ sDepartment of Surgery, Kaplan Hospital, Rehovot, Israel
}

Received 26 August 1996; accepted 13 March 1997

\section{ABSTRACT}

Successful application of anticancer therapy, and especially photodynamic therapy (PDT) mediated by type II (PDTII) processes, depends on the oxygen content within the tumor before, during and after treatment. The high consumption of oxygen during type II PDT imposes constraints on therapy strategies. Although rates of oxygen consumption and repletion during PDTII were suggested by theoretical studies, direct measurements have not been reported. Application of a novel oxygen sensor allowed continuous and direct in situ measurements (up to a depth of 8-9 $\mathrm{mm}$ from the tumor surface and for several hours) of temporal variations in the oxygen partial pressure $\left(\mathrm{pO}_{2}\right)$ during PDT. Highly pigmented M2R mouse melanoma tumors implanted in CD1 nude mice were treated with bacteriochlorophyll-serine (Bchl-Ser; a new photodynamic reagent) and were subjected to fractionated illumination (700 $<\lambda<900 \mathrm{~mm})$ at a fluence rate of $12 \mathrm{~mW} \mathrm{~cm}^{-2}$. This illumination led to total oxygen depletion with an average consumption rate of 7.2 $\mu M\left(\mathrm{O}_{2}\right) \mathrm{s}^{-1}$. Spontaneous reoxygenation (at an average rate of $\left.2.5 \mu M\left(\mathrm{O}_{2}\right) / \mathrm{s}\right)$ was observed during the following dark period. These rates are in good agreement with theoretical considerations (Foster et al., Radiat. Res. 126, 296, 1991 and Henning et al., Radiat. Res. 142, 221, 1995). The observed patterns of oxygen consumption and recovery during prolonged periods of light/dark cycles were interpreted in terms of vasculature damage and sensitizer clearance. The presented data support the previously suggested advantages of fractionated illumination for type II photodynamic processes.

\footnotetext{
*To whom correspondence should be addressed at: Department of Biochemistry, The Weizmann Institute of Science, Rehovot 76100, Israel. Fax: 972-8-934-4118;

e-mail: bcscherz@weizmann.weizmann.ac.il

(C) 1997 American Society for Photobiology 0031-8655/97 $\$ 5.00+0.00$
}

\section{INTRODUCTION}

In type II photodynamic therapy (PDTII) $\dagger(1,2)$, a photosensitizing drug, light and oxygen are used to cause photochemically induced cell death. Singlet oxygen molecules $\left({ }^{\prime} \mathrm{O}_{2}\right)$ are generated via energy transfer from sensitizing dye molecules that are administered to the tumor and then excited by either visible (VIS) or near infrared (NIR) radiation $(2,3)$. Tumor destruction during PDTI is believed to be achieved by in situ generation of ${ }^{\prime} \mathrm{O}_{2}$ and the consequent oxidation of cellular constituents (4-6). However, the $\mathrm{O}_{2}$ distribution in tumor tissues is often heterogeneous $(7,8)$. Cells in hypoxic tumor regions are thought to be more resistant to PDTII, as well as to radio- and chemotherapies $(1,4,9)$. Furthermore, extensive photodynamic activity near the blood capillaries causes a temporary reduction of the oxygen concentration at remote tumor zones, which further limits the tumor response to PDTII $(10,11)$. Thus, information on the temporal and spatial oxygen levels in tumors is important for selecting the therapy strategy and predicting its efficiency.

Theoretical studies have related the rate of oxygen depletion with a particular light dose, spectral characteristics and concentration of the applied photosensitizer. Almost linear relationships were predicted between the initial concentration and depletion time of oxygen in the tumor tissue (10) (Figs. 1 and 2). Following these studies, it should take $\sim 12$ $s$ to reduce the oxygen concentration in a tumor tissue to $100 \mu M$ by providing light at $50 \mathrm{~mW} \mathrm{~cm}^{-2}$ to hematoporphyrin derivative (HPD; 5-10 $\mu \mathrm{g} / \mathrm{g}$ treated tissue). Completion of tumor reoxygenation (at a rate of $1-4 \mu \mathrm{M} / \mathrm{s}$ ) is expected to take 30-60 s (11). According to these studies, shorter dark periods (approaching continuous illumination), higher fluence rates and/or longer illumination times would reduce the efficiency of PDTII, especially in zones that are fairly remote from the tumor vascular bed. Significantly higher rates of animal survival after PDT with fractionated

\footnotetext{
$\dagger$ Abbreviations: Bchl-Ser, bacteriochlorophyll-serine; HPD, hematoporphyrin derivative; NIR, near infrared; ${ }^{1} \mathrm{O}_{2}$, singlet oxygen: PDT, photodynamic therapy; PDTII, type II PDT; $\mathrm{pO}_{2}$, oxygen partial pressure; VIS, visible.
} 

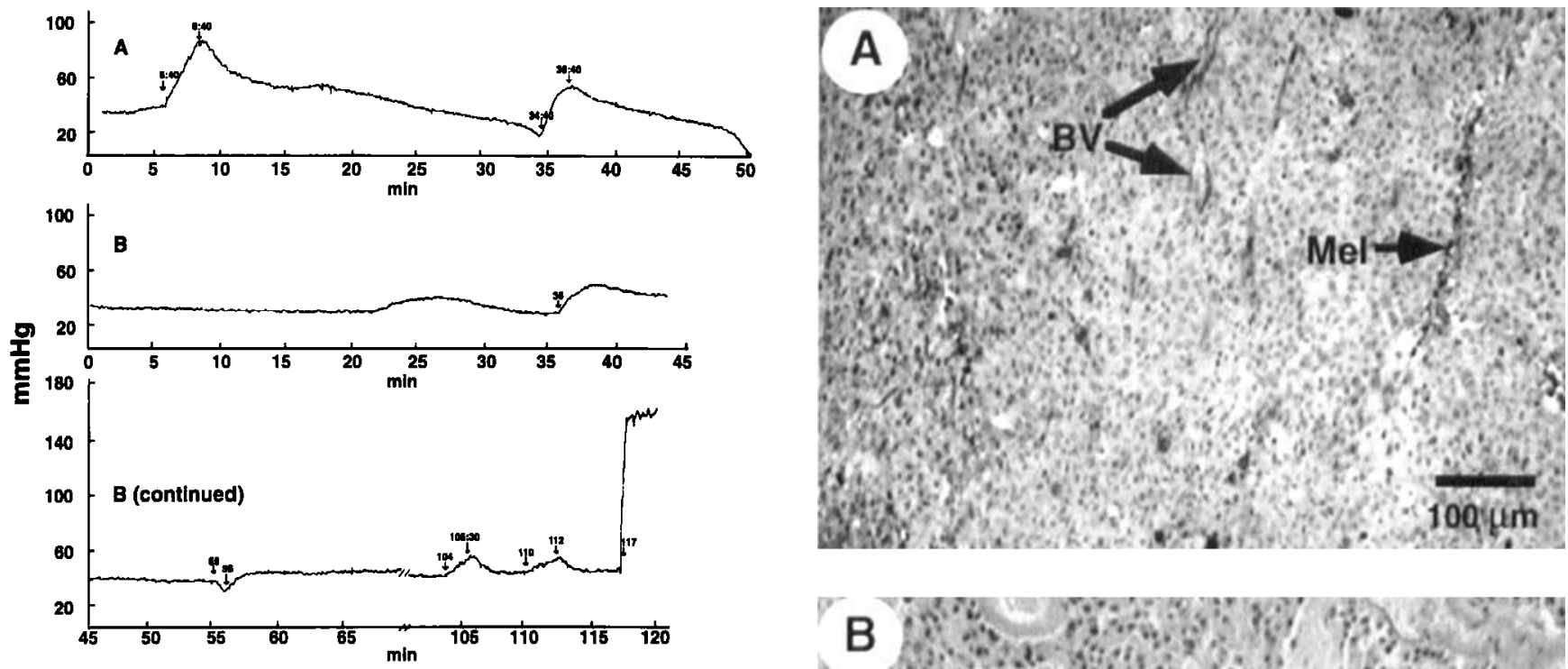

Figure 1. In vivo measurement of $\mathrm{pO}_{2}$ and the dynamic response of the oxygen microsensor. The $\mathrm{pO}_{2}$ in striated muscle (Psoas) (A) and subcutaneous tissue (B) is depicted. In panel $A$, the mouse was ventilated at $\mathrm{t}=6: 40$ and $34: 40$ with $100 \% \mathrm{O}_{2}$ for $2 \mathrm{~min}$ by using a ventilation mask. In panel $\mathrm{B}$, spontaneous increase in tissue $\mathrm{pO}_{2}$, concomitant with movements of the anesthetized animal, was noticed at $t=36: 00 \mathrm{~min}$. Therefore, the mouse was reanesthetized at $t=39: 00 \mathrm{~min}$. In panel $\mathbf{B}$ (continued), the mouse was ventilated with $4 \%$ oxygen for $1 \mathrm{~min}$ at $\mathrm{t}=55: 00 \mathrm{~min}$, and with $100 \%$ oxygen for 1.5 and $2 \mathrm{~min}$ at $t=104: 00$ and $110: 00 \mathrm{~min}$, respectively. At $t$ $=117 \mathrm{~min}$, the experiment was terminated and the sensor tip pulled out of the tumor and allowed to equilibrate with air. Pariels $B$ and $B$ (continued) depict data obtained from the same mouse.

rather than continuous illumination (of similar total energy) experimentally supported these claims (10-12).

Quantitation of the tumor oxygen content during the course of PDT was attempted in several studies (13-15). Application of an indirect method (utilizing the transcutaneous Clark electrode) for follow-up of tumor oxygen levels enabled the calculation of oxygen consumption rates of $\sim 10^{-2}-10^{-3} \mu \mathrm{M} \mathrm{O}_{2} / \mathrm{s}$ in Photofrin II-based PDT (15). This value is two to three orders of magnitude less than predicted $(10,11)$. The precision and time resolution of this indirect and similar techniques for monitoring the kinetics of oxygen consumption during PDT were low due to the inherent limitations of polarographic assays. For example, oxygen consumption by the electrode and the electrical current involved in polarographic techniques result in low accuracy of measurements, especially at low oxygen tensions where the signal-to-noise ratio is low. Furthermore, the stability of the electrode could be affected by contamination (protein poisoning) (14). The use of transcutaneous electrodes requires heating of the skin surface to $44^{\circ} \mathrm{C}$ in order to accelerate oxygen diffusion, which imposes an undesirable impact of the probe itself. The dimensions of these electrodes limit their spatial resolution. Cumulatively, these shortcomings confine the application of polarographic assays to subcutaneous tumors (15). Other methods for measuring tissue oxygen and related parameters consist of more complex procedures that usually involve analyses of tissue biopsies (14).

The drawbacks of existing methodologies for in vivo determination of oxygen concentrations in tumors during

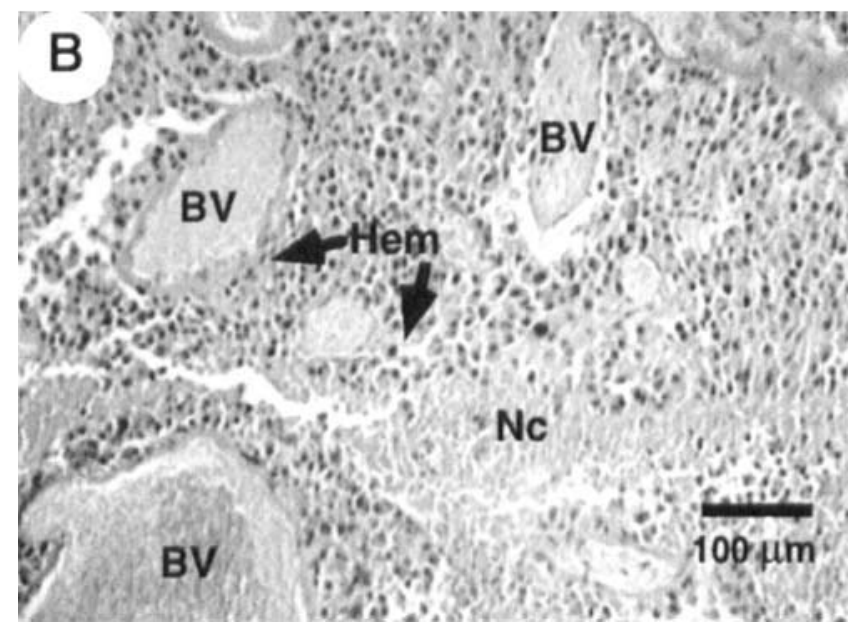

Figure 2. Photodynamic damage induced by Bchl-Ser-based PDT of solid M2R melanoma tumors. Histological sections of untreated (A) and treated ( $24 \mathrm{~h}$ after treatment; B) tumors. Visualization was achieved by light microscopy of hematoxylin/eosin/light green staining of the tumor sections. BV, blood vessel; Hem, hemorrhage formation; Nc, necrotic regions; Mel, melanotic cells.

PDTII prompted the search for in situ measurements. An optical sensor, developed by us to monitor oxygen partial pressure $\left(\mathrm{pO}_{2}\right)$ in blood $(16,17)$ seemed suitable for performing similar measurements in solid tissues. When inserted into blood or tissue, this optical-sensing fiber continuously probes $\mathrm{pO}_{2}$ by measuring the fluorescence of pyrene immobilized on a solid support, which is quenched in the presence of $\mathrm{O}_{2}$. This probe can be used to monitor even low local concentrations of oxygen $\left(1 \mathrm{mmHg}\right.$ ). Temporal changes in $\mathrm{pO}_{2}$ are immediately sensed with a high time resolution (seconds). Furthermore, this sensor does not consume oxygen and is protected from poisoning $(16,17)$.

Herein, this novel sensor was utilized to monitor the oxygen levels in implanted M2R mouse melanoma tumors during PDT. Such fully pigmented tumors cannot be treated by Photofrin II and other VIS-red-absorbing sensitizers because the red light beam utilized with these sensitizers is absorbed and scattered to a great extent by the melanin-a phenomenon that limits the depth of Photofrin sensitization to 1-3 $\mathrm{mm}$. In order to enable treatment at deeper locations, a new photosensitizer, bacteriochlorophyll-serine (Bchl-Ser), was 
synthesized (18-21). This photosensitizer is a water-soluble molecule that strongly absorbs in the NIR (extinction coefficient of $10^{5} \mathrm{M} / \mathrm{cm}$ at $\lambda \max =770-780 \mathrm{~nm}$ ) and delivers high photocytotoxicity ( $\sim 200$ times that of Photosan, a hematoporphyrin-based drug) in cell cultures (21). Bacteriochlorophyll-serine exhibits low dark toxicity, fast clearance rates from healthy animal tissues and slower clearance rates from tumor tissues (half-life times of 2 and $15 \mathrm{~h}$, respectively) (21). These properties enable delivery of photodynamic activity throughout the entire volume of large solid tumors. The therapeutic ability of Bchl-Ser in PDT was tested and found to be efficient in the animal tumor model described below (21-24) with 34 of 40 animals completely cured to date (Zilberstein et al., in preparation).

\section{MATERIALS AND METHODS}

\section{Photosensitizer}

Bacteriochlorophyll-serine was prepared from the natural pigment, bacteriochlorophyll-a, that was chemically modified at the $\mathrm{C}-17$ propionic residue of its macrocycle and conjugated to a serine molecule (18-21). The Bchl-Ser was kept dry under argon in the dark at $-20^{\circ} \mathrm{C}$ until used.

\section{Tumor model}

The M2R mouse melanoma cells were maintained as monolayers, as previously described (25). For tumor implantation, the cells were scraped off the plates, suspended in normal saline and subcutaneously injected $\left(1 \times 10^{6}\right.$ cells $\left./ 25 \mu \mathrm{L}\right)$ into the backs of CDI male $(30-35 \mathrm{~g})$ nude mice, as previously described $(21,26)$. By $\sim 4-5$ weeks, the melanotic tumors appeared as dark, solid and confined structures with an average weight of $4-6 \mathrm{~g}$.

\section{Anesthesia}

Mice were anesthetized by intraperitoneal injections ( $40 \mu \mathrm{L})$ of ketamine (5 mg/kg; Rhone Merieux, Lyon, France) and xylazine (1 $\mathrm{mg} / \mathrm{kg}$; Bayer, Leverkusen. Germany). This cocktail has a minimal effect on blood flow and, consequently, little effect on the oxygen levels within the treated tissue. The applied dose was sufficient to keep the mice anesthetized for $-30-40 \mathrm{~min}$. Additional injections $(10-20 \mu \mathrm{L})$ were performed as needed to maintain anesthesia until the conclusion of the treatment (up to $8 \mathrm{~h}$ ).

\section{Administration of drug}

The Bchl-Ser $(200 \mu \mathrm{g} / 50 \mu \mathrm{L}$ of alcohol [95\%]/saline [1:1]) was administered to anesthetized mice by direct injection into the tumor using a $28 \times 1 / 2$ gauge needle. In oxygen measurements, the drug was injected close to the site of the microsensor.

\section{Light irradiation}

(a) In light-dependent oxygen depletion experiments, a home-built light source (equipped with a $100 \mathrm{~W}$ white halogen lamp, RG-700 cut-off filter and $10 \mathrm{~cm}$ water layer filter) provided radiation at 700 $<\lambda<900 \mathrm{~nm}$ with an average power of $12 \mathrm{~mW} \mathrm{~cm}^{-2}$ and was fractionated as described in the individual experiments. The irradiating light was controlled by an optical lens and the illuminated spot on the tissue (diameter $1.2 \mathrm{~cm}$ ) covered the examined area of the tumor. Detrimental effects due to heating (edema and/or skin injury) were not observed at the site of illumination. (b) In PDT experiments, the same light source and settings were used, except that the RG filter was removed, the average power was $100 \mathrm{~mW} \mathrm{~cm}^{-2}$, the light was emitted continuously for $30 \mathrm{~min}$ and the light spot covered the entire tumor.
Table 1. The $\mathrm{pO}_{2}$ levels in tissues and implanted $\mathrm{M} 2 \mathrm{R}$ melanoma tumors in nude mice

\begin{tabular}{lc}
\hline \multicolumn{1}{c}{ Tissue } & $\mathrm{pO}_{2}(\mathrm{mmHg})$ \\
\hline Spleen & $22 \pm 1(\mathrm{n}=2)^{*}$ \\
Kidney & $22 \pm 2(\mathrm{n}=4)$ \\
Striated muscle & $37 \pm 2(\mathrm{n}=5) \dagger$ \\
Subcutaneous tissue & $40 \pm 2(\mathrm{n}=4)$ \\
M2R melanoma turnor & $0-30(\mathrm{n}=30)$ \\
\hline
\end{tabular}

$*_{\mathrm{n}}=$ Number of animals.

†Measurements were conducted in five mice: Psoas two and Rectus abdominis three.

\section{Histopathology}

Mice were sacrificed by cervical dislocation at appropriate stages of the photodynamic treatment, and the tumor was removed and placed in Buene's fixative. Following a fixation of $24 \mathrm{~h}$, the tumor was paraffin-embedded, sectioned $(10 \mu \mathrm{m})$ and the sections stained with hematoxylin/eosin with light green as previously described (26). Pathological differences between samples were assessed by light microscopy (Optiphot-2, Nikon, Japan).

\section{Oxygen measurements}

Oxygen microsensor. The Blood Gas Monitor (developed by OST, Kiryat Bialik, Israel) is an on-line, real-time system that provides continuous monitoring of blood oxygen with a time response that is diffusion limited. This oxygen sensor monitors the fluorescence of a pyrene derivative, which is quenched in a concentration-dependent manner by molecular oxygen. The pyrene molecules are attached to the porous tip of a fused silica fiber $(180 \mu \mathrm{m}$ diameter $)(16,17)$. The porous glass tip is coated with a thin gold layer, which insulates the sensor from interference by external light and is covered with an anticoagulant membrane that prevents clot formation upon contact with blood. Measurements of blood oxygen levels with this sensor are accurate and reliable (17).

Calibration. Prior to each experiment, the sensor was calibrated for $\mathrm{pO}_{2}$ measurements in the range of $28-57 \mathrm{mmHg}$ by using distilled water equilibrated at $37^{\circ} \mathrm{C}$ with oxygen : nitrogen mixtures $(4$, 8 and $100 \% \mathrm{O}_{2}$ ). At the end of each experiment, the oxygen sensor was placed in the calibration solution to ensure readout stability during the examination period.

In vivo oxygen measurements. The oxygen sensor was inserted a few millimeters into the desired tissue (kidney, spleen, striated muscle [Rectus abdominis or Psoas], subcutaneous tissues or tumor) of an anesthetized mouse, through a hypodermic needle (22 gauge). Oxygen levels were recorded under dark conditions until a stable $\mathrm{pO}_{2}$ baseline was established. This baseline value was used as the normal tissue $\mathrm{pO}_{2}$. In all experiments, the tumor was exposed to fractionated illumination for a few minutes prior to drug administration; this was done to assess the response of the sensor and the tumor to light. The Bchl-Ser was then injected directly into and allowed to spread in the tumor for $1-2 \mathrm{~h}$. The site of injection was selected so as to be in proximity of the site of $\mathrm{pO}_{2}$ measurement. The tumor was then exposed to fractionated illumination for different time intervals. During the entire examination period, the $\mathrm{pO}_{2}$ levels were continuously displayed on the computer screen as $\mathrm{mmHg}$. Unless specified, measurements were carried out under normal atmospheric conditions. When indicated, a ventilation mask was used to provide the mice with $4 \%$ or $100 \%$ oxygen.

\section{RESULTS}

\section{$\mathrm{pO}_{2}$ in healthy and tumor tissues}

In vivo $\mathrm{pO}_{2}$ in solid tissues (kidney, spleen, striated muscle and subcutaneous tissue) of 15 mice and M2R tumors of 30 mice was measured with the oxygen sensor (Table 1). The recorded $\mathrm{pO}_{2}$ levels in the healthy tissues were reproducible 


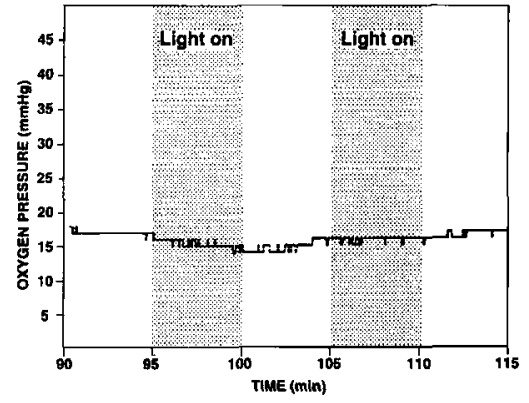

Figure 3. Effect of light alone on the in vivo $\mathrm{pO}_{2}$ in an $\mathrm{M} 2 \mathrm{R}$ melanoma tumor. The sensor was inserted into the tumor at $t=0$, and at $\mathrm{t}=95$ and $105 \mathrm{~min}$ the tumor was illuminated for $5 \mathrm{~min}$. The light intervals are indicated by the dashed areas.

and characteristic of the different organs. In contrast, $\mathrm{pO}_{2}$ values for the tumors varied significantly, which suggested site dependence.

The dynamic response range of the oxygen sensor was demonstrated by monitoring the $\mathrm{pO}_{2}$ levels in two striated muscle sites: Rectus abdominis and Psoas. The $\mathrm{pO}_{2}$ levels in these sites were similar and around $39 \mathrm{mmHg}$ (Fig. 1A, Table 1). Upon exposure of the mouse to $100 \% \mathrm{O}_{2}(\mathrm{t}=6$ : $40 \mathrm{~min}$ ), the tissue $\mathrm{pO}_{2}$ increased almost instantaneously and reached $\sim 88 \mathrm{mmHg} 2 \mathrm{~min}$ later. At this time the mouse was reexposed to normal atmosphere, which resulted in an initial rapid decrease of tissue $\mathrm{pO}_{2}$, followed by a slow decline to a level lower than the initial baseline. This low reading seemed indicative of a respiration/perfusion failure. Despite reventilation with $100 \%$ oxygen for 2 min (starting at $\mathrm{t}=$ $34: 40 \mathrm{~min}$ ), which increased the $\mathrm{pO}_{2}$ from $\sim 18$ to $56 \mathrm{mmHg}$, the respiration/perfusion failure was apparently irreversible and the animal died $15 \mathrm{~min}$ later. Concomitantly, the oxygen sensor showed a steady decline to zero. The cause of death was probably a combination of mechanical and oxygen toxicity apnea that often occurs in spontaneously breathing anesthetized or very young animals and also in human neonates $(27,28)$.

Correlation between tissue $\mathrm{pO}_{2}$ and the state of anesthesia of the mouse is exemplified in Fig. 1B. Under the skin of this anesthetized mouse, the baseline $\mathrm{pO}_{2}$ level was $32-40$ $\mathrm{mmHg}$ during a period of $35 \mathrm{~min}$. When a sudden increase in the $\mathrm{pO}_{2}$ level $(t=36 \mathrm{~min})$, accompanied by movements of the anesthetized animal, was observed, a bolus of the anesthetics was administered. This slowly redeepened the level anesthesia, which caused a decline to baseline tissue $\mathrm{pO}_{2}$ levels. When the mouse was transferred to a $4 \% \mathrm{O}_{2}$ atmosphere for $1 \mathrm{~min}(\mathrm{t}=56 \mathrm{~min})$, the tissue $\mathrm{pO}_{2}$ immediately declined and reached a minimum of $31 \mathrm{mmHg}$. Subsequently, the $\mathrm{pO}_{2}$ level overshot the baseline and stayed at $44 \mathrm{mmHg}$ for the next $\mathbf{4 2} \mathrm{min}$. When the mouse was later exposed twice to $100 \% \mathrm{O}_{2}$, for only 1.5 and $2 \mathrm{~min}$, a rapid but transient increase in the tissue $\mathrm{pO}_{2}$ occurred. Upon termination of the in vivo measurement, the sensor was removed, allowing spontaneous equilibration with room air $\left(\mathrm{pO}_{2}, 155 \mathrm{mmHg}\right)$. These results demonstrated the ability of the sensor to determine $\mathrm{pO}_{2}$ in normal solid tissues and demonstrated its dynamic behavior.

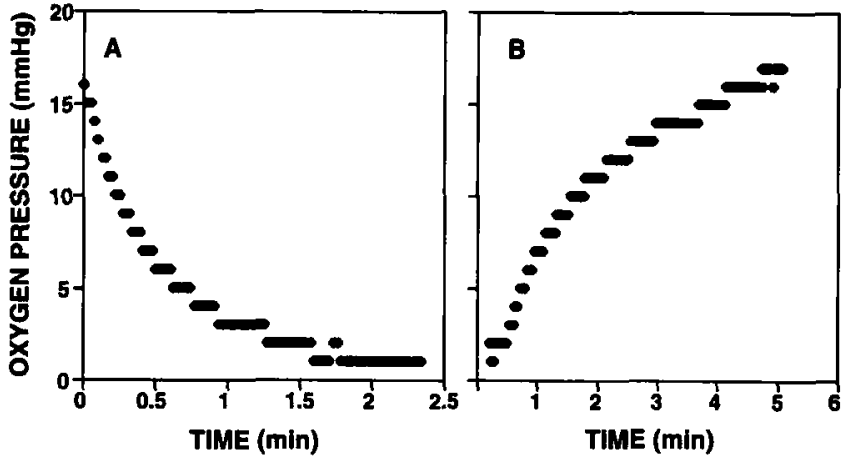

Figure 4. In vivo oxygen depletion and reoxygenation profiles during the light/dark cycles of a Bchl-Ser-treated melanoma tumor. Typical oxygen depletion (A) and reoxygenation (B) profiles obtained during the first cycles of fractionated illumination of a BchlSer-treated M2R tumor. Data (dots) fitted with single exponential functions (Eqs. 1 and 2) with correlations of 0.994 for depletion and 0.993 for reoxygenation. Time zero refers to the start of the light (A) and dark (B) periods. The vertical and horizontal resolutions of the instrument are $1 \mathrm{mmHg}$ and $0.9 \mathrm{~s}$, respectively.

\section{Photodynamic damage induced in melanoma tumors by Bchl-Ser and light}

The damage induced in solid $\mathrm{M} 2 \mathrm{R}$ melanoma tumors treated by Bchl-Ser and light was determined histologically (Fig. 2). Untreated tumor tissue (Fig. 2A) consisted primarily of densely packed malignant cells $(\mathrm{MC})$ with occasional melanotic areas (Mel) and well-defined blood vessels (BV) that were easily visualized when longitudinally displayed. Twenty four hours after exposure to Bchl-Ser and light, tissue destruction accompanied by significant vasodilation, hemorrhage $(\mathrm{Hem})$ and necrosis $(\mathrm{Nc})$ were observed in the treated tumors (Fig. 2B). Administration of light alone or of Bchl-Ser with no subsequent illumination was without effect (data not shown).

\section{$\mathrm{pO}_{2}$ during fractionated illumination of tumors treated with Bchl-Ser}

Following anesthesia of an M2R tumor-bearing mouse, the oxygen sensor was inserted into the tumor $(t=0 \mathrm{~min})$ and the $\mathrm{pO}_{2}$ levels were recorded until a stable baseline around $20 \mathrm{mmHg}$ was established. Illumination of the tumor prior to drug administration had no effect on the $\mathrm{pO}_{2}$ level (Fig. 3 ). This control was routinely performed before each experiment to ensure that the sensor is light impenetrable. The temporal response of tumor oxygen levels to Bchl-Ser and fractionated illumination was examined for a few hours in 30 M2R tumor-bearing mice. Various patterns of individual light/dark cycles (after Bchl-Ser administration) prompted respective oxygen depletion and repletion within seconds to minutes that appeared similar in all animals (Figs. 4-7).

Typical oxygen depletion and repletion profiles during the early light/dark cycles are depicted in Fig. 4A and B and were best fit by

$$
\mathrm{p}\left[\mathrm{O}_{2}\right]_{\mathrm{t}}=\mathrm{A}+\mathrm{B} \times \exp \left(-k_{\mathrm{D}} \mathrm{t}\right)
$$

for the period of illumination and by

$$
\mathrm{p}\left[\mathrm{O}_{2}\right]_{\mathrm{t}}=(\mathrm{A}-\mathrm{B}) \times \exp \left(-k_{\mathrm{R}} \mathrm{t}\right)+\mathrm{B}
$$

for the period of oxygen recovery in the dark (with corre- 


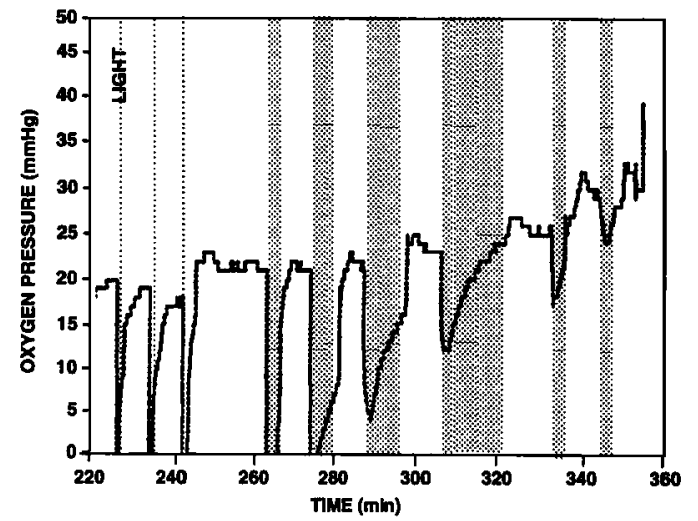

Figure 5. The in vivo $\mathrm{pO}_{2}$ in $\mathrm{M} 2 \mathrm{R}$ mouse melanoma under PDT conditions (pattern 1). Starting from the time of the sensor insertion $(\mathrm{t}=0 \mathrm{~min})$, the $\mathrm{pO}_{2}$ was continuously monitored in the implanted tumor. At $\mathrm{t}=106 \mathrm{~min}$, Bchl-Ser was administered into the tumor and the mouse kept in the dark for about $2 \mathrm{~h}$. At $\mathrm{t}=26 \mathrm{~min}$, the light source was turned "on" for $20 \mathrm{~s}$. Thereafter, the light was turned "on" and "off" at the indicated times. The light intervals are indicated by the dashed lines.

lations of 0.994 and 0.993 , respectively). The time (t) is given in seconds, $k_{\mathrm{D}}$ and $k_{\mathrm{R}}$, the rate constants for oxygen depletion and repletion averaged from 10 different experiments are $0.03 \pm 5 \times 10^{-3}$ and $0.009 \pm 3 \times 10^{-4} \mathrm{mmHg}$ $\mathrm{s}^{-1}$, respectively. $\mathrm{A}$ and $\mathrm{B}$ depend on the initial $\mathrm{pO}_{2}$ level in each experiment. For the particular experiment depicted in Fig. 4, the parameters $A$ and $B$ equal 0.8 and $15 \mathrm{mmHg}$, respectively. Usually it takes $\sim 100 \mathrm{~s}$ for complete depletion (where $\left[\mathrm{pO}_{2}\right]_{t \geq 100}=0 \pm 1 \mathrm{mmHg}$ ) and $360 \mathrm{~s}$ for the complete repletion of the oxygen content in the tumor tissue (Fig. 4).

To calculate the corresponding average rates of light-dependent oxygen consumption and tumor reoxygenation (in the dark), $K_{\mathrm{c}}$ and $K_{\mathrm{o}}$, respectively, we have made the following assumptions: (a) the blood flow rate is $0.025 \mathrm{~mL} /(\mathrm{min}$ $\left.\mathrm{cm}^{2}\right)(29,30)$; (b) the photodynamic action of Bchl-Ser consumes both the soluble and hemoglobin-bound oxygen fractions; (c) during the dark period the bound and soluble oxygen are recovered to their preillumination levels; (d) hypoxia imposed by the photodynamic process increases the arteriovenous oxygen extraction from 5 to $15 \%(\mathrm{vol} / \mathrm{vol})$ of the total blood supply (15).

During the $6 \mathrm{~min}$ dark period (Fig. 4B), $0.025 \times 6 \mathrm{~mL}$ of blood flow into the tumor, providing $15 \mu \mathrm{L}$ of oxygen to 1 $\mathrm{g}$ tumor tissue. This corresponds to $600 \mu M$ oxygen that is consumed during the $108 \mathrm{~s}$ of illumination. Oxygen molecules carried into the tumor by the blood during this time $(180 \mu M)$ are also consumed. Hence, the rate of oxygen consumption is approximately $7.2 \mu M\left[\mathrm{O}_{2}\right] / \mathrm{s}$ and that of reoxygenation amounts to $2.5 \mu M\left[\mathrm{O}_{2}\right] / \mathrm{s}$.

Comparison of these values with those given in the literature for oxygen consumption due to photofrin-based PDT has to consider the different pigment concentrations in the tumor tissue and the actual light absorbed by the sensitizer at the applied light domains (10). An accurate number cannot be provided at the moment because we did not measure the pigment concentration within the tumor at the time of irradiation. However, starting with $200 \mu \mathrm{g}$ of Bchl-Ser $/ 5 \mathrm{~g}$ tumor tissue and assuming half-life time of $2.5 \mathrm{~h}$ for the

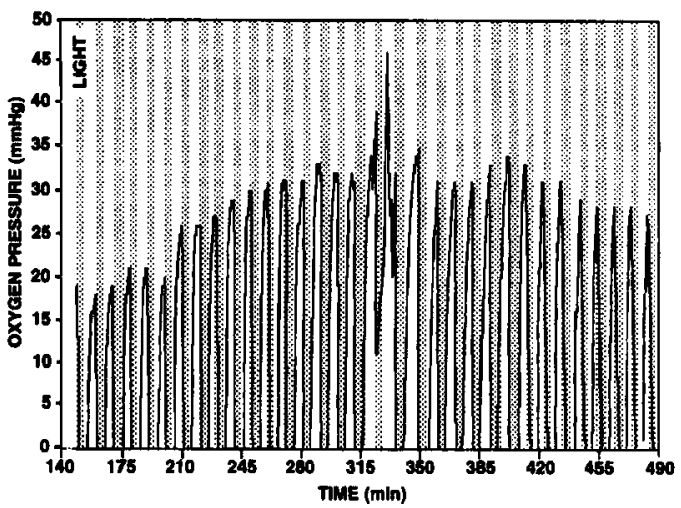

Figure 6. The long-term in vivo $\mathrm{pO}_{2}$ in $\mathrm{M} 2 \mathrm{R}$ mouse melanoma under PDT conditions (pattern 2). Sensor inserted at $t=0$, BchlSer administrated at $\mathrm{t}=20 \mathrm{~min}$ and fractionated illumination $\sim 5$ min light/ -5 min dark started at $t=146$ min. The light intervals are indicated by the dashed areas.

retention of Bchl-Ser and photochemically active metabolites (21), we may estimate that the Bchl-Ser concentration at the time of irradiation was approximately $30 \mu M$ compared to $4 \mu M$ Photofrin considered in the theoretical (10) and other experimental studies (15). On the other hand, the fluence rate used in the current experiments is four times smaller than the one used by these other groups. Note that the ratio between the time of depletion and complete repletion of oxygen $(\sim 1: 3)$ is larger than found by Tromberg et al. (15) (1:2) but smaller than predicted by Henning et al. (11) $(1: 6)$.

The long-term response that developed within a few hours of illumination displayed three different patterns of behavior. This phenomenon was further examined in 12 of the tumorbearing animals studied. The first pattern, showing an eventual loss of the oxygen depletion effect (Fig. 5) was observed in four animals. The sensor was inserted into the tumor at $t$ $=0 \mathrm{~min}$. The Bchl-Ser was administered at $\mathrm{t}=106 \mathrm{~min}$, and $2 \mathrm{~h}$ later $(\mathrm{t}=226 \mathrm{~min})$ the tumor was illuminated for the first time. This first illumination of the tumor, even if for only $20 \mathrm{~s}$, which was in the presence of Bchl-Ser, induced a prompt depletion of the tumor oxygen levels to 5$8 \mathrm{mmHg}$. During the ensuing dark period, the $\mathrm{pO}_{2}$ was completely replenished to preillumination baseline levels $(\sim 30$ $\mathrm{mmHg}$ ). This was followed by the administration of various illumination periods and intervals between illuminations as indicated. During the first few light/dark cycles $(226<t<$ $275 \mathrm{~min}$ ), oxygen levels dropped to nearly zero upon illumination, which indicated a high efficiency of oxygen consumption. However, upon additional exposures, the oxygen depletion due to exposure to light became progressively smaller. At $t>275 \mathrm{~min}$, the postillumination oxygen levels overshot the baseline values and progressively increased. A correlation between oxygen depletion and illumination was observed only in the presence of Bchl-Ser, which suggests that interaction of the drug and light was necessary for attenuation of the tumor $\mathrm{pO}_{2}$.

In a second pattern of behavior (Fig. 6), followed in four mice, we observed temporal variations in the tumor oxygen levels although the oxygen depletion/repletion effect was kept throughout the entire irradiation time. The tumor, injected with Bchl-Ser was exposed to light/dark cycles of $\sim 5$ 


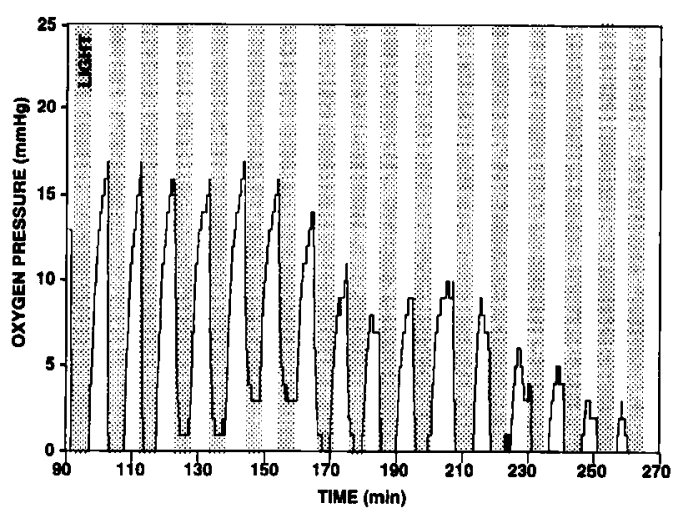

Figure 7. The long-term in vivo $\mathrm{pO}_{2}$ in $\mathrm{M} 2 \mathrm{R}$ mouse melanoma under PDT conditions (pattern 3). Sensor inserted at $t=0$, BchlSer administrated at $t=15 \mathrm{~min}$ and fractionated illumination $\sim 5$ min light $\sim 5$ min dark started at $t=95 \mathrm{~min}$. The light intervals are indicated by the dashed areas.

min each. The $\mathrm{pO}_{2}$ baseline in the dark periods increased steadily ( $146<\mathrm{t}<300 \mathrm{~min}$ ), then appeared to stabilize for several hours, and later displayed a slight decline $(300<\mathrm{t}$ $<490 \mathrm{~min}$ ). With this protocol of exposure to light (alternating periods of $\sim 5$ min light and dark), a third pattern (Fig. 7) was also observed in four mice. Here, an eventual depletion of the entire oxygen content was observed. During the early dark intervals ( $95<\mathrm{t}<160 \mathrm{~min}$ ), the tumor $\mathrm{pO}_{2}$ levels consistently returned to the preirradiation baseline levels. However, with increasing time $(160<\mathrm{t}<270 \mathrm{~min})$, the $\mathrm{pO}_{2}$ baseline during the dark intervals progressively declined, and as it approached zero, there was an attenuation of the response to light. Complete depletion of oxygen, as a result of illumination (Figs. 6 and 7), indicated that the BchlSer concentration was in excess throughout the experiment.

The depth to which the light penetrates into the tumor was estimated by controlled insertion of the sensor into the tumor. Light-dependent oxygen consumption could be observed by insertion of the sensor up to a depth of $\sim 8-9 \mathrm{~mm}$ from the skin.

\section{DISCUSSION}

This study examined the spatial and temporal variations in the concentration of oxygen within solid tumors, especially the rate of oxygen depletion and repletion during PDT. While homeostatic $\mathrm{pO}_{2}$ values were previously measured by polarographic techniques, reports on direct measurement of temporal oxygen variations in situ and with sufficient hightime resolution were lacking. Application of a newly developed optical sensor enabled the execution of such in vivo measurements at a time resolution of $<1 \mathrm{~s}$, comparison of the recorded data with theoretical considerations and the proposal of a light regimen for effective PDT.

Our continuous measurements in situ of the homeostatic oxygen pressure in normal and tumor tissues (Table 1) are in good agreement with previous polarographic measurements $(31-38)$. The observed variations in $\mathrm{pO}_{2}$ within a particular tumor and between tumors of different animals not subjected to PDT (Table 1) are probably due to the heterogenous morphology of these solid tumors. This heterogeneity of tumor $\mathrm{pO}_{2}$ was previously attributed to variations in vascular density and uneven distribution of regions containing proliferating, quiescent and/or necrotic zones $(24,26,30)$.

The rapid and total depletion of local oxygen upon illumination of tumors that contained saturating concentrations of sensitizers was previously predicted $(10,11)$. In fact, following application of Bchl-Ser and subsequent illumination, the measured tumor $\mathrm{pO}_{2}$ values dropped rapidly, often to below instrumental resolution (Figs. 5-7), and returned to baseline level only after the light was turned off. Exposure to light alone or application of Bchl-Ser alone did not affect the $\mathrm{pO}_{2}$ in solid $\mathrm{M} 2 \mathrm{R}$ tumors. The in vivo light-dependent oxygen consumption as mediated by Bchl-Ser suggests its potential to deliver PDTII in a manner similar to chlorophyll-serine which has been demonstrated by us to generate ${ }^{\prime} \mathrm{O}_{2}$ in the presence of light in vitro (39).

Herein, the rates of oxygen consumption $\left(K_{\mathrm{c}}\right)$ and reoxygenation $\left(K_{\mathrm{o}}\right)$ in the tumor tissue were calculated to be 7.2 and $2.5 \mu M\left(\mathrm{O}_{2}\right) / \mathrm{s}$, respectively. Comparison with literature data (predicted and experimental) relies on the knowledge of intratumor pigment concentration, light absorbance and incident irradiation. While the estimate of the Bchl-Ser concentration and the incident irradiation are fairly reasonable, it is difficult to have a good estimate of the light absorbance within the tumor. Still, the observed $K_{\mathrm{c}}$ and $K_{\mathrm{o}}$ are in the range of values predicted by Foster et al. (10) and Henning et al. (11). The calculated rates for this system are significantly higher than the corresponding rates suggested by Tromberg et al. based on indirect measurements with transcutaneous Clark electrodes (15). The $K_{\mathrm{c}} / K_{\mathrm{o}}$ in this study is $\sim 3$, whereas Tromberg et al. (15) suggested a value of 2 .

Although the short-term $\mathrm{pO}_{2}$ response to illumination after administration of Bchl-Ser was similar in all the animals tested (Figs. 4-7), the long-term response could follow one of at least three patterns of behavior that are probably related to development of vascular damage as exemplified in Fig. 2B. In this experiment, spillage of blood into the damaged area and hemorrhage formation due to vascular injury may culminate in clot formation and blood stasis with consequent impact on local $\mathrm{pO}_{2}$ distribution $(4,40)$. In one pattern, a progressive decline in the effect of light on $\mathrm{pO}_{2}$ and a slight increase in the baseline oxygen levels in the dark occurs (Fig. 5). A simple mathematical manipulation shows that both the amount and rate of oxygen depletion are proportional to the sensitizer concentration in the treated zone (Eqs. A2 and A3 in Foster et al. (10)). Thus, a decrease in the extent of oxygen depletion observed in this pattern should reflect a decrease in the concentration of Bchl-Ser within the irradiated zone. This phenomenon could be explained by pigment degradation and photobleaching within the irradiated zone, as well as by fast tissue and vascular clearance due to the high water solubility of Bchl-Ser. If the latter is indeed correct, then the clearance rate for Bchl-Ser from the tumor under illumination $\left(\mathrm{t}_{1 / 2} \approx 2.5 \mathrm{~h}\right.$ ) would be significantly higher than that reported earlier by us for M2R tumors in unilluminated mice (21). Both the fast clearance of Bchl-Ser and the increase in oxygen concentrations during dark periods might be related to local vascular damage. The slow, but steady, increase in the $\mathrm{pO}_{2}$ baseline during dark periods (Fig. 5) may be indicative of decreased metabolism, which accompanies photodynamic cell destruction. Furthermore, the spillage of blood into the damaged area may temporally 
elevate $\mathrm{pO}_{2}$ to levels existing in adjacent blood vessels (Fig. 2B). At times relatively late after illumination of the BchlSer-treated tumor region, moderate oxygen repletion started even during the light period (Fig. 5, t $>270 \mathrm{~min}$ ). In this case, the local concentration of Bchl-Ser probably became the rate-limiting factor for light-dependent oxygen consumption, but due to some pigment redistribution into the site of measurement the process did not totally stop. Indeed when illumination was terminated, the rate of oxygen repletion was immediately enhanced due to elimination of the competition for oxygen by the residual photodynamic process. Further diffusion of residual Bchl-Ser into the examined site enabled brief depletion spikes in response to light at each light/dark cycle. The magnitude of these spikes became progressively smaller, apparently reflecting the trend of BchlSer clearance.

The other two patterns of long-term behavior of tissue $\mathrm{pO}_{2}$ during fractionated illumination of Bchl-Ser-treated tumors (Figs. 6 and 7) probably reflect on the vascular density within or near the examined zone. In both, complete oxygen depletion during illumination is observed throughout the entire experiment. However, while one is characterized by complete oxygen recovery in the dark to a relatively constant baseline (Fig. 6), in the other there is a progressive decline of baseline $\mathrm{pO}_{2}$ in the dark that approaches zero (Fig. 7). For complete oxygen recovery to occur, the inspected zone must be within $160 \mu \mathrm{m}$ of active capillaries $(10,11)$. Such a situation is probable in a tumor site that is rich in vasculature. In that case, a low fluence rate of illumination, such as that used in the present study, will insult only part of the capillary bed and continuous oxygen supply will be ensured by the remaining undamaged part. In contrast, if the monitored zone is positioned at a tumor site with poor vasculature, damage to the capillaries may result in complete discontinuation of the oxygen supply.

Similar long-term patterns were reported previously using the transcutaneous Clark electrodes (15). However, the short-term pattern observed here allows relation of these patterns with the tumor microenvironment in real time. Yet, the suggested explanations for these patterns are awaiting verification by complementary methodologies, such as magnetic resonance imaging.

In conclusion, under conditions where the oxygen concentration is the rate-limiting factor of PDTI, optimal oxygen consumption can probably be achieved by fractionated illumination at a moderate fluence rate, where the light delivery is synchronized with the oxygen recovery. Our data support the hypothesis that an alternate light/dark cycle provides optimum oxygen consumption that eventually may lead to optimal PDT effect $(10,11)$. However, the light and dark durations depend upon the concentration and absorbance of the photosensitizer as well as upon the light fluence rate.

Acknowledgements-Y.S. is the incumbent of the Charles and Tillie Lubin Chair of Biochemical Endocrinology. This study was performed in partial fulfillment of J.Z.'s Ph.D. thesis for the Feinberg Graduate School, The Weizmann Institute of Science. We thank Dr. V. Brumfield for skillful help, OST for providing the oxygen sensor instrument, Ms. B. Schick for reviewing the manuscript and Ms. R. Benjamin for her excellent secretarial assistance. This study was supported in part by grants from the Lynne and William Frankel Fund for the
Diagnosis and Treatment of Ovarian and Breast Cancer, the Jaffe Family Foundation, Mrs. S. Zuckerman, Yeda Research and Development Co. Ltd. (Israel), the Israeli Ministry of Science and Technology and the Commission of the European Communities.

\section{REFERENCES}

1. Henderson, B. W. and V. H. Fingar (1989) Oxygen limitation of direct tumor cell kill during photodynamic treatment of a murine tumor model. Photochem. Photobiol. 49, 299-304.

2. Foote, C. S. (1987) Light activated pesticides. Am. Chem. Soc. Symp. Ser. 1, 22-38.

3. Dougherty, T. J. (1992) Photochemistry in the treatment of cancer. Adv. Photochem. 17, 275-311.

4. Henderson, B. W. and T. J. Dougherty (1992) How does photodynamic therapy work? Photochem. Photobiol. 55, 145-157.

5. Keene, J. P., D. Kessel, E. J. Land, R. W. Redmond and T. G. Truscott (1986) Direct detection of singlet oxygen sensitized by haematoporphyrin and related compounds. Photochem. Photobiol. 43, 117-120.

6. Weishaupt, K. R., C. J. Gomer and T. J. Dougherty (1976) Identification of singlet oxygen as the cytotoxic agent in photoinactivation of a murine tumor. Cancer Res. 36, 2326-2329.

7. Vaupel, P., K. Schlenger, C. Knoop and M. Hockel (1991) Oxygenation of human tumors: evaluation of tissue oxygen distribution in breast cancers by computerized $\mathrm{O}_{2}$ tension measurements. Cancer Res. 51, 3316-3322.

8. Lartigau, E., L. Vitu, M. C. Haie, M. F. Cosset, M. Delapierre, A. Gerbaulet, F. Eschwege and M. Guichard (1992) Feasibility of measuring oxygen tension in uterine cervix carcinoma. Eur. J. Cancer 28, 1354-1357.

9. Hirst, D. G. (1989) Tumor hypoxia implication for therapy. Int. J. Radiat. Biol. 56, 207-213.

10. Foster, T. H., R. S. Murant, R. G. Bryant, R. S. Knox, S. L. Gibson and R. Hilf (1991) Oxygen consumption and diffusion effects in photodynamic therapy. Radiat. Res. 126, 296-303.

11. Henning, J. P., R. L. Fournier and J. A. Hampton (1995) A transient mathematical model of oxygen depletion during photodynamic therapy. Radiat. Res. 142, 221-226.

12. Veenhuizen, R. B. and F. A. Stewart (1995) The importance of fluence rate in photodynamic therapy: is there a parallel with ionizing radiation dose-rate effects? Radiother. Oncol. 37, 131135.

13. Reed, M. W., A. P. Mullins, G. L. Anderson, F. N. Miller and T. J. Wieman (1989) The effect of photodynamic therapy on tumor oxygenation. Surgery 106, 94-99.

14. Stone, H. B., J. M. Brown, T. L. Phillips and R. M. Sutherland (1993) Oxygen in human tumors: correlations between methods of measurement and response to therapy. Radiat. Res. 136, 422434.

15. Tromberg, B. J., A. Orenstein, S. Kimel, S. J. Barker, J. Hyatt, J. S. Nelson and M. W. Berns (1990) In vivo tumor oxygen tension measurements for the evaluation of the efficiency of photodynamic therapy. Photochem. Photobiol. 52, 375-385.

16. Zilberstein, J., A. Bromberg and G. Berkovic (1994) Fluorescence study of pyrene chemically bound to controlled-pore glass. J. Photochem. Photobiol. A Chem. 77, 69-81.

17. Bromberg, A., J. Zilberstein, S. Riesemberg, I. E. Benor, E. Silberstein, J. Zimnavoda, G. Frishman and A. Krietzman (1996) Optical fiber sensors for blood gases and $\mathrm{pH}$, based on porous glass tips. Sensor and Actuators B 31, 181-191.

18. Fiedor, L. (1994) Modified chlorophylls as models for primary photosynthesis and photosensitizers for photodynamic therapy of cancer. Ph.D. thesis, Weizmann, Institute of Science, Rehovot, Israel.

19. Scherz, A., Y. Salomon and L. Fiedor (1994) Chlorophyll and bacteriochlorophyll derivatives, preparation and pharmaceutical compositions comprising them as photosensitizers for photodynamic therapy. Chem. Abst. 120, 386.

20. Scherz, A., Y. Salomon and L. Fiedor (1994) Preparation of chlorophyll conjugates and their application as photosensitizers in photodynamic therapy and general diagnostics. YEDA, Weizmann Institute of Science, European published patent application no. EP0584552. 
21. Rosenbach-Belkin, V., L. Chen, L. Fiedor, I. Tregub, F. Pavlotsky, V. Brumfeld, Y. Salomon and A. Scherz (1996) Serine conjugates of chlorophyll and bacteriochlorophyll: phototoxicity in vitro and tissue distribution in mice bearing melanoma tumors. Photochem. Photobiol. 64, 174-181.

22. Zilberstein, J., A. Scherz, A. Bromberg, P. Bendel, M. Neeman and Y. Salomon (1994) Chlorophyll based PDT of melanoma: follow up by MRI and by tissue inserted oxygen sensor. In The Tumor Microenvironment: Its Characterization. Modification and Clinical Implication. Faculty of Medicine, University of Granada, Granada, Spain. [Abstract]

23. Zilberstein, J., A. Scherz, A. Bromberg, P. Bendel, M. Neeman and Y. Salomon (1995) Mechanisms involved in chlorophyll based photoinduced cell damage: photodynamic therapy of melanoma. Proc. Soc. Magnet. Reson. 3, 1681. [Abstract]

24. Zilberstein, J., A. Scherz, A. Bromberg, P. Bendel, M. Neeman and Y. Salomon (1996) The effects of bacteriochlorophyll based PDT on tumor vasculature. Proc. Soc. Magnet. Reson. 2, 1099. [Abstract]

25. Gerst, J. E., J. Sole, J. P. Mather and Y. Salomon (1986) Regulation of adenylate cyclase by $\beta$-melanotropin in M2R melanoma cell line. Mol. Cell. Endocrinol. 46, 137-147.

26. DeJordy, J. O., P. Bendel, A. Horowitz, Y. Salomon and $H$. Degani (1992) Correlation of MR imaging and histologic findings in mouse melanoma. J. Magnet. Reson. Imag. 2, 695-700.

27. Alvaro, R., J. Alvarez, K. Kwiatkowski, D. Cates and H. Rigatto (1994) Induction of mixed apneas by inhalation of $100 \%$ oxygen in preterm infants. J. Appl. Physiol. 77, 1666-70.

28. Tobin, J. and A. Jubran (1995) Oxygen takes the breath away: old sting, new setting. Mayo Clin. Proc. 70, 403-404.

29. Guyton, A. C. (1981) Textbook in Medical Physiology, 6th ed. W. B. Saunders Co. Philadelphia, PA.

30. Vaupel, P. W. (1994) Blood flow, oxygenation, tissue pH distribution, and bioenergetic status of tumors. Ernst Schering Res. Found. Lect. 23, 79.

31. Gustafsson, U., F. Sjoberg, D. H. Lewis and P. Thorborg (1994) Effect of hypoxic hypoxia and ritanserin on capillary flow and oxygenation in rabbit skeletal muscle. Acta Physiol. Scand. 150, $39-45$.

32. Harrison, D. K., M. Kessler and S. K. Knauf (1990) Regulation of capillary blood flow and oxygen supply in skeletal muscle in dogs during hypoxaemia. J. Physiol. (Lond.) 420, 431-446.

33. Dewhirst, M. W., E. T. Ong. B. Klitzman, T. W. Secomb, R. Z. Vinuya, R. Dodge, D. Brizel and J. F. Gross (1992) Perivascular oxygen tensions in a transplantable mammary tumor growing in a dorsal flap window chamber. Radiat. Res. 130, 171182.

34. Khandelwal, S. R., R. S. Randad, P. S. Lin, H. Meng, R. N. Pittman, H. A. Kontos, S. C. Choi, D. J. Abraham and U. R. Schmidt (1993) Enhanced oxygenation in vivo by allosteric inhibitors of hemoglobin saturation. Am. J. Physiol. 265, H1450H1453.

35. Brezis, M., S. N. Heyman and F. H. Epstein (1994) Determinants of intrarenal oxygenation. II. Hemodynamic effects. Am. J. Physiol. 267, F1063-F1068.

36. Brezis, M., Y. Agmon and F. H. Epstein (1994) Determinants of intrarenal oxygenation. I. Effects of diuretics. Am. J. Physiol. 267, F1059-F1062.

37. Gatenby, R. A., H. B. Kessler, J. S. Rosenblum, L. R. Coia, P. J. Moldofsky, W. H. Hartz and G. J. Broder (1988) Oxygen distribution in squamous cell carcinoma metastases and its relationship to outcome of radiation therapy. Int. J. Radiat. Oncol. Biol. Phys. 14, 831-838.

38. Pappova, N., E. Siracka, A. Vacek and J. Durkovsky (1982) Oxygen tension and prediction of the radiation response. Polarographic study in human breast cancer. Neoplasma 29, 669674.

39. Fiedor, L., A. A. Gorman, I. Hamblett, V. Rosenbach-Belkin, Y. Salomon, A. Scherz and I. Tregub (1993) A pulsed laser and pulse radiolysis study of amphiphilic chlorophyll derivatives with PDT activity toward malignant melanoma. Photochem. Photobiol. 58, 506-11.

40. van Geel, I., H. Oppelaar, P. F. Rijken, H. J. Bernsen, N. E. Hagemeier, D. K. A. van, O. R. H and F. A. Stewart (1996) Vascular perfusion and hypoxic areas in RIF-1 tumours after photodynamic therapy. Br. J. Cancer 73, 288-293. 